\title{
Qualidade de água de uma lagoa rasa em meio rural no sul do Brasil
}

\author{
Raquel W. Cunha' ${ }^{1}$, Manoel D. N. Garcia Jr. ${ }^{1}$, Edélti F. Albertoni ${ }^{1}$ \& Cleber Palma-Silva ${ }^{1}$
}

\section{RESUMO}

No seu entorno, a lagoa Caiubá apresenta regiões preservadas e áreas com cultura de arroz e de pecuária. Esta pesquisa descreve o padrão de variação sazonal e espacial das variáveis fisico-químicas e biológicas durante os anos de 2009 e 2010, em nove pontos distribuídos espacialmente na lagoa, representando suas partes norte, central e sul. A qualidade da água foi avaliada através da aplicação do índice de estado trófico (IET), do índice de qualidade de água (IQA) e das classes da Resolução CONAMA 357/2005. Foram coletadas amostras de invertebrados bentônicos e determinados os Índices Bióticos (IB) de acordo com os modelos da Environmental Protection Agency (EPA-USA) e a adaptação do Biological Monitoring Working Party (BMWP). Os resultados evidenciaram um sistema com alta turbidez e conteúdo de fósforo, além de baixa razão N:P, e com poucas alterações sazonais e espaciais nas variáveis medidas. Embora os resultados do IQA e CONAMA tenham classificado a lagoa Caiubá como águas de excelente a boa qualidade, os resultados do IET e dos IB foram opostos classificando a lagoa como sistema alterado e poluído. Conclui-se que esses índices devam ser readequados através de avaliações sistemáticas visando refletir as condições de qualidade de ecossistemas aquáticos em meio rural, na região subtropical do Brasil.

Palavras-chave: rizicultura, índice biótico, IET, IQA, CONAMA 357

\section{Water quality of a shallow lagoon in rural area in Southern Brazil}

\begin{abstract}
The Caiubá lagoon has its surroundings with preserved areas and areas with rice cultivation and livestock. This study describes the pattern of seasonal and spatial variation of physico-chemical and biological variables during the years 2009 and 2010 at nine points, representing part of northern, central and southern regions. Water quality was evaluated by applying the trophic state index (TSI) and the water quality index (WQI), and classes according to CONAMA Resolution 357/2005. Samples of benthic invertebrates were collected and determined the biotic index (BI), following the models of the Environmental Protection Agency (EPA-USA), and adaptation of Biological Monitoring Working Party (BMWP). The results demonstrated a system with high turbidity and phosphorus content, and low N:P ratio, and with little seasonal and spatial changes in the measured variables. Although the results of WQI and the CONAMA have classified the lagoon waters with excellent to good quality, the results of the TSI and the BI were opposites, classifying the ecosystem as amended and polluted system. It is concluded that these indices should be readjusted through systematic evaluations in order to reflect the quality condition of aquatic ecosystems in rural areas in the subtropical region of Brazil.
\end{abstract}

Key words: rice cultivation, biotic index, TSI, WQI, CONAMA 357 


\section{INTRODUÇÃO}

A planície costeira do estado do Rio Grande do Sul é rica em recursos hídricos; seu processo de formação com influência das transgressões e regressões marinhas do período quaternário formou depressões e terras planas, com corpos de água interligados por arroios e grandes extensões de terras alagadas. Essas terras férteis têm sido intensamente utilizadas para desenvolvimento agropecuário, com pecuária de corte $\mathrm{e}$, mais recentemente, com a expansão da cultura do arroz irrigado.

Os corpos de água na região são caracterizados, ecologicamente, como lagos rasos, que são sensíveis às variações naturais ou antrópicas devido à sua pequena profundidade, podendo apresentar grande variação espacial e temporal das suas características físicas e químicas ao longo do ano (Scheffer, 1998; Trindade et al., 2009; Zambrano et al., 2009). Este tipo de ecossistemas é frágil frente à poluição antrópica em que o principal fator que interfere na resiliência do ecossistema é a carga externa de nutrientes, sobretudo o fósforo (Scheffer, 1998; Janse et al., 2008); desta forma, o uso do solo no entorno tem influência direta no estado ecológico do ambiente aquático.

Por apresentar as quatro estações do ano bem definidas, o clima subtropical pode promover variações das características físicas, químicas e biológicas dos lagos ao longo do ano e, consequentemente, causar alterações no metabolismo do ecossistema e na sua biodiversidade. Estudos nesses ambientes são importantes para detectar tais variações, sendo informações imprescindíveis para futuros planos de gestão de recursos hídricos localizados nessas regiões (Coveney et al., 2005; Zhu et al., 2007).

$\mathrm{O}$ estudo da qualidade de água em lagos teve início no século passado utilizando-se variáveis como estratificação térmica e de oxigênio, distribuição de larvas de Chironomidae (insetos dípteros) e concentração de nutrientes. A partir desses trabalhos pioneiros muitas tentativas de caracterizar a tipologia e a qualidade dos ambientes vêm sendo desenvolvidas principalmente em regiões temperadas, com a criação de índices que sumarizem o estado trófico e a qualidade de água (Carlson, 1977). Para a região tropical e subtropical tem-se buscado, mais recentemente, adaptações desses índices de forma a caracterizar os ecossistemas de acordo com as regiões climáticas distintas (Mercante \& Tucci-Moura, 1999; Lamparelli, 2004).

Além das informações sobre o metabolismo abiótico do sistema, outra ferramenta que reflete com boa acuracidade a qualidade de água, é a utilização dos chamados índices bióticos utilizando-se organismos aquáticos classificados conforme sua tolerância a fatores ambientais. Vários desses índices foram desenvolvidos principalmente nas regiões temperadas e para rios e riachos (Rosemberg \& Resh, 1993). Para a região subtropical algumas adaptações têm sido desenvolvidas, por exemplo, a modificação do Biological Monitoring Working Party (BMWP) adaptada para o Brasil pela Secretaria do Meio Ambiente do estado do Paraná e por Junqueira \& Campos (1998) para rios de Minas Gerais, utilizado com sucesso pela CETEC (Fundação Centro Tecnológico de Minas Gerais).

O objetivo deste estudo é caracterizar a variação sazonal e espacial das variáveis físicas, químicas e biológicas no espaço de um ano, abrangendo períodos de produção agrícola de arroz e avaliar e discutir a qualidade de suas águas através do Índice de Qualidade da Água (IQA), das classes do CONAMA (2005), do índice do estado trófico (IET) e pela aplicação de índices bióticos utilizando-se invertebrados bentônicos.

\section{Material e Métodos}

$\mathrm{O}$ estudo foi conduzido na lagoa Caiubá $\left(32^{\circ} 22^{\prime} \mathrm{S}\right.$ e $52^{\circ}$ $30^{\prime}$ W), localizada na Planície Costeira do Rio Grande do Sul, no município de Rio Grande (Figura 1). O clima na região é o subtropical úmido (Cfa, segundo a classificação de KöppenGeiger). A lagoa faz parte do Sistema Hidrológico do Taim e ao Sul da lagoa Caiubá está localizada a Estação Ecológica do Taim, distante cerca de $2 \mathrm{~km}$ com o objetivo de preservar a fauna e a flora regional.

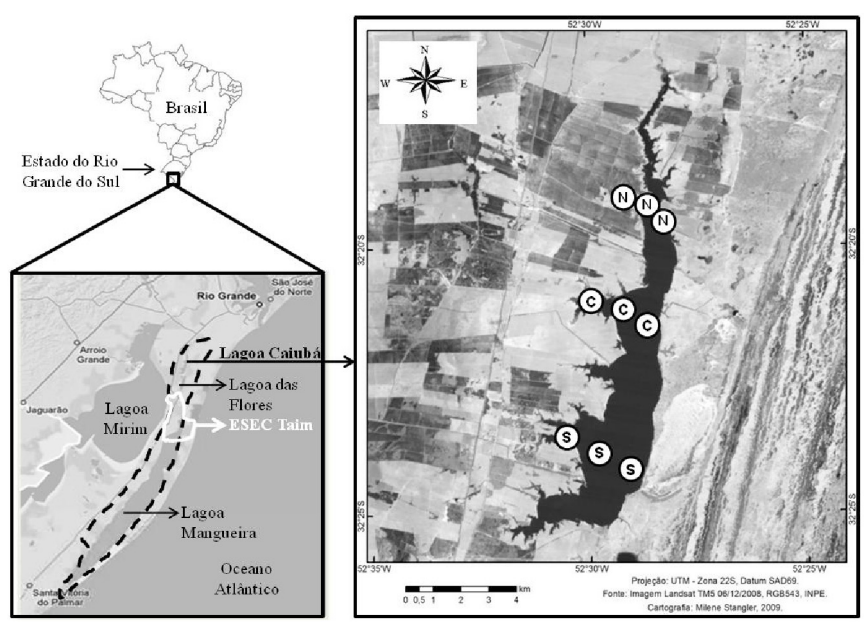

Figura 1. Localização do Sistema Hidrológico do Taim (delimitado pela linha tracejada), da Estação Ecológica do Taim (ESEC Taim), da lagoa Caiubá e dos pontos de amostragem na sua região Norte $(\mathrm{N})$, Central $(\mathrm{C})$ e Sul (S)

A lagoa Caiubá tem conformação alongada, com aproximadamente $8,3 \mathrm{~km}$ de comprimento e $1,2 \mathrm{~km}$ de largura, com aproximados $30 \mathrm{~km}^{2}$ de área superficial. A área de entorno em 2005 é classificada com ocupação sendo de $10,4 \%$ de cultura de arroz, $8,9 \%$ de pastagem, $19,3 \%$ de solos sem cobertura vegetal, $25,6 \%$ de campos arenosos e dunas e $11,3 \%$ de banhados, entre outros (Alba et al., 2012). Sua região norte possui profundidade média de $2,27 \mathrm{~m}$ e distância entre as margens oeste a leste de $0,8 \mathrm{~km}$, tornando-se mais profunda e larga ao sul, onde apresenta $2,86 \mathrm{~m}$ de profundidade média e distância entre as margens de $3,5 \mathrm{~km}$ (dados dos autores).

As amostragens ocorreram entre Dezembro de 2009 e Novembro de 2010 sendo realizadas duas coletas por estação do ano, totalizando 8 amostragens. Foram definidos nove pontos amostrais em 3 regiões da lagoa: Norte (prevalência de campos de arroz no entorno), Central (presença de campos de arroz, pecuária e alguns banhados nas margens) e Sul (presença de agropecuária e dunas nas margens) com três pontos em cada região, localizados próximo às margens Oeste e Leste e da região limnética. 
Em cada ponto foram coletados os dados físicos, químicos e biológicos da água superficial $(20 \mathrm{~cm})$. Em campo foram determinados a temperatura da água, condutividade, $\mathrm{pH}$, profundidade e transparência pelo disco de Secchi. Uma vez por estação do ano foi medido o perfil vertical da temperatura e do oxigênio dissolvido nos pontos na região limnética da lagoa.

Em laboratório foram determinados a turbidez, o oxigênio dissolvido, a alcalinidade, o material em suspensão e sólidos dissolvidos totais, a clorofila-a, o nitrogênio, o fósforo total e fosfato, o nitrato, nitrito, sulfato e cloreto, $\mathrm{DBO}_{5}$ e coliformes termotolerantes; foram calculadas, também, a razão N:P e a extensão da zona eufótica a partir do valor da transparência da água.

Dados meteorológicos (temperatura média do ar, umidade relativa, velocidade e direção do vento, taxa e dias de precipitação e taxa de evaporação) foram obtidos durante o período amostrado, pela Estação Meteorológica Principal de Rio Grande, operada pelo Laboratório de Meteorologia da Universidade Federal do Rio Grande (FURG) distante aproximadamente $45 \mathrm{~km}$ da lagoa Caiubá.

Os invertebrados bentônicos foram coletados com amostrador tipo Kajak, nas três regiões da lagoa, em três repetições, totalizando 27 amostras. As amostras foram fixadas com álcool 80\% corado com Rosa de Bengala e em laboratório lavadas em água corrente sob peneira de $250 \mu \mathrm{m}$; em seguida, o material foi separado em microscópio estereoscópico e os indivíduos contados e identificados até o menor nível taxonômico possível.

Foi calculada, para cada ponto, a riqueza de táxons e comparadas sua composição e abundância com ANOVA oneway. Aos resultados foram aplicados os três índices bióticos: aquele utilizado pela Environmetal Protection Agency (EPA) para os Estados Unidos e o índice BMWP, com adaptações da Secretaria de Meio Ambiente e Recursos Hídricos do Paraná (www.meioambiente.pr.gov.br) e de Junqueira \& Campos (1998), utilizado pela Fundação Centro Tecnológico de Minas Gerais - CETEC, no Brasil, ambos possuem categorizações em classes equivalentes à qualidade de água, conforme a Tabela 1 .
Para a análise sazonal das variáveis da água foi realizada a média dos nove pontos amostrais em duas coletas, totalizando um $\mathrm{n}=18$ para cada estação do ano; a análise espacial das variáveis foi realizada por estação do ano utilizando-se a média dos três pontos em cada região amostrada na lagoa, $\operatorname{com} u m \mathrm{n}=6$.

Realizou-se uma comparação entre as três regiões de coleta $(\mathrm{n}=12)$ e as estações do ano $(\mathrm{n}=18)$ com ANOVA one-way (dados $\log (\mathrm{x}+1)$ transformados) e o teste de Tukey foi aplicado para comparação post hoc. Diferenças entre as médias das variáveis foram consideradas significativas quando $\mathrm{p}<0,05$. Uma ordenação com o método de Ward utilizando a distância Euclidiana foi aplicada para verificar a relação entre as variáveis ao longo do ano. Todos os testes foram realizados com o software Past versão 2.15 (Hammer et al., 2001).

A qualidade da água da lagoa Caiubá foi determinada com índices de estado trófico (IET), índice de qualidade da água (IQA) e a resolução 357/2005 do CONAMA. O Índice de Estado Trófico (IET) utilizado foi o proposto por Carlson (1977), com as modificações de Mercante \& Tucci-Moura (1999) $\left(\mathrm{IET}_{\mathrm{M}}\right)$ e por Lamparelli (2004) $\left(\mathrm{IET}_{\mathrm{L}}\right)$. Este índice utiliza os valores médios anuais de clorofila-a, fósforo total e transparência do disco de Secchi. Para o índice geral é feita, na adaptação de Lamparelli (2004) uma média simples desses parâmetros. Neste trabalho foi utilizada a média simples dos valores de clorofila e fósforo total, conforme recomendado pela autora para locais com altas taxas de turbidez inorgânica. Na adaptação de Mercante \& Moura (1999) se utilizam, também, os valores de fósforo solúvel reativo (FSR) calculando-se o índice geral através de uma equação com médias ponderadas. Para enquadramento do estado de qualidade de água utilizou-se a seguinte classificação (Lamparelli, 2004) com as respectivas equivalências dos valores de fósforo total, transparência e clorofila-a, tal como a equivalência com os valores de enquadramento do IET pela adaptação de Mercante \& Moura (1999) (Tabela 2).

O Índice de qualidade da água (IQA) utilizado foi o proposto pela National Sanitation Foundation modificado pelo COMITESINOS (1990). A determinação do IQA é obtida pelo produtório ponderado correspondente a 8 parâmetros (i)

Tabela 1. Valores dos índices bióticos e as respectivas classes de qualidade de água segundo os critérios da Secretaria de Meio Ambiente e Recursos Hídricos do estado do Paraná, Environmental Protection Agency (EPA) e Fundação Centro Tecnológico de Minas Gerais (CETEC)

\begin{tabular}{cclccl}
\hline Classes & Paraná & Qualidade de água & EPA & CETEC & Qualidade de água \\
I & $>150$ & Otima & $\leq 4,5$ & $\geq 86$ & Excelente \\
II & $121-150$ & Boa & & $64-85$ & Boa \\
III & $101-120$ & Aceitável & $4,5-6,5$ & $37-63$ & Satisfatória \\
IV & $61-100$ & Duvidosa & $6,5-8,5$ & $17-36$ & Ruim \\
V & $35-60$ & Poluída & & & Muito ruim \\
VI & $16-35$ & Muito poluída & $\geq 8,5$ & $\leq 16$ & Sistema fortemente alterado \\
VII & $<16$ & Fortemente poluída & & & \\
\hline
\end{tabular}

Tabela 2. Categorização do estado trófico segundo as modificações de Mercante \& Moura (1999) (IET M $_{\text {) Lamparelli }}$ (2004) (IET ), com os valores correspondentes de transparência do disco de Secchi, fósforo total (P-total) e clorofila-a

\begin{tabular}{|c|c|c|c|c|c|}
\hline Estado trófico & Valores - IET & Valores - IET $_{M}$ & Secchi - S (m) & P-total (mg L') & Clorofila-a $\left(\mu \mathrm{g} \mathrm{L^{-1 } )}\right.$ \\
\hline Ultra oligotrófico & $\mathrm{IET} \leq 47$ & & $S \geq 2,4$ & $P \leq 0,008$ & $\mathrm{CL} \leq 1,17$ \\
\hline Mesotrófico & $52<\mathrm{IET} \leq 59$ & $44<\mathrm{IET}>54$ & $1,7>S \geq 1,1$ & $0,019<P \leq 0,052$ & $3,24<C L \leq 11,03$ \\
\hline Supereutrófico & $63<\mathrm{IET} \leq 67$ & & $0,8>S \geq 0,6$ & $0,120<P \leq 0,233$ & $30,55<\mathrm{CL} \leq 69,05$ \\
\hline Hipereutrófico & IET $>67$ & & $0,6>S$ & $0,233<P$ & $69,05<\mathrm{CL}$ \\
\hline
\end{tabular}


calculado a partir da qualidade relativa (q) de cada parâmetro, entre 0 e 100 , obtido da respectiva "curva média de variação de qualidade", em função de sua concentração ou medida e do peso relativo $(\mathrm{w})$ de cada parâmetro, um número entre 0 e 1 , atribuído em função da sua importância para a conformação global de qualidade $\left(\right.$ IQA $=$ Пqi $\left.{ }^{\text {wi }}\right)$ (COMITESINOS, 1990).

A partir do cálculo efetuado determina-se a qualidade das águas brutas numa escala que varia de 0 a 100: $0<$ IQA $<25$ - muito ruim; $26<$ IQA $<50$ - ruim; $51<$ IQA $<70-$ regular; $71<$ IQA $<90$ - bom e $91<$ IQA $<100$ - excelente (COMITESINOS, 1990).

Segundo o CONAMA, a Classificação foi obtida pela aplicação das classes de água regidas pela resolução 357/2005. Os parâmetros analisados e os limites para determinação de cada Classe estão especificados na Tabela 3, com exceção da Classe Especial, que é voltada para a preservação e conservação e devem ser mantidas as condições originais da água. A Classe 4 só estabelece valores para o pH e o oxigênio dissolvido e alguns dos outros parâmetros, que são determinados só até a Classe 3, apresentaram valores acima. Para classificar essas variáveis foi incluída, neste trabalho, uma nova classe chamada " $>$ Classe 3" (maior do que a Classe 3). Os resultados da classificação do CONAMA foram expressos como frequência de ocorrência nas Classes para cada uma das variáveis analisadas, procedimento este utilizado com o objetivo de valorizar o conjunto de dados disponíveis e considerar a relevância de eventuais alterações de qualidade observadas.

\section{Resultados E Discussão}

As médias sazonais dos dados meteorológicos $(\mathrm{n}=3)$ durante o período amostrado são apresentadas na Tabela 4. De forma geral, as análises comparativas demonstraram as diferenças esperadas em função do padrão sazonal das características climáticas subtropicais, com menores temperaturas no inverno e maior velocidade do vento e precipitação nos meses de verão. Constatou-se que o Outono, embora com a segunda taxa de precipitação durante o ano, apresentou a menor profundidade da coluna de água provavelmente como resultado da utilização de água durante o Verão para irrigação das lavouras de arroz. O padrão de mistura polimítico com ausência de estratificação
Tabela 4. Dados médios meteorológicos para as estações do ano durante o período de estudo

\begin{tabular}{lcccc}
\hline \multicolumn{1}{c}{ Parâmetro } & Verão & Outono & Inverno & Primavera \\
Temperatura média do ar $\left({ }^{\circ} \mathrm{C}\right)$ & $23,8 \mathrm{~A}$ & $20,0 \mathrm{AB}$ & $13,3 \mathrm{C}$ & $17,3 \mathrm{BC}$ \\
Umidade relativa (\%) & $83,4 \mathrm{~A}$ & $80,4 \mathrm{~A}$ & $83,6 \mathrm{~A}$ & $75,4 \mathrm{~A}$ \\
Velocidade do vento $\left(\mathrm{m} \mathrm{s}^{-1}\right)$ & $3,4 \mathrm{~A}$ & $2,6 \mathrm{AB}$ & $2,2 \mathrm{~B}$ & $2,9 \mathrm{AB}$ \\
Direção do vento & $\mathrm{L} / \mathrm{SE}$ & $\mathrm{NE} / \mathrm{SE}$ & $\mathrm{NE} / \mathrm{S} 0$ & $\mathrm{NE} / \mathrm{S} / \mathrm{SE}$ \\
Taxa de precipitação $(\mathrm{mm})$ & $157,1 \mathrm{~A}$ & $127,1 \mathrm{~A}$ & $117,4 \mathrm{~A}$ & $76,3 \mathrm{~A}$ \\
Dias de precipitação & $11,0 \mathrm{AB}$ & $16,0 \mathrm{~A}$ & $12,0 \mathrm{AB}$ & $8,0 \mathrm{~B}$ \\
Taxa de evaporação $(\mathrm{mm})$ & $126,1 \mathrm{~A}$ & $115,1 \mathrm{~A}$ & $80,7 \mathrm{~A}$ & $127,3 \mathrm{~A}$ \\
\hline
\end{tabular}

Fonte: Estação Meteorológica Principal de Rio Grande da Universidade Federal do Rio Grande Letras diferentes representam com diferença significativa (ANOVA, $p<0,05$ )

térmica da coluna de água, foi constante durante o período estudado, sendo típico de lagos rasos costeiros, conforme Scheffer (1998); não ocorreu déficit de oxigênio em nenhum dos períodos analisados.

A Tabela 5 apresenta o padrão de variações sazonais para as variáveis da água medidas durante o período de estudo, agrupadas sazonalmente.

A associação das variáveis medidas entre as estações do ano demonstrou agrupamento entre variáveis relacionadas. Os nutrientes, assim como DBO, turbidez, condutividade e alcalinidade apresentaram uma relação mais próxima associada com a profundidade de Secchi (Figura 2). Agrupamento semelhante também foi encontrado por Lamparelli (2004) para ambientes lênticos do estado de São Paulo.

Apenas as variáveis cloreto, nitrito, profundidade e condutividade elétrica não tiveram diferença ao longo do ano. Todas as outras diferiram pelo menos entre duas estações do ano. Entre as regiões (norte, central e sul) foram detectadas somente pequenas variações; assim, os resultados de caracterização são discutidos levando-se em conta a variação anual do ecossistema. De forma geral foram registrados, no verão, os maiores valores de transparência, clorofila-a, nitrito, sulfato e DBO.

Não ocorreu diferença entre as taxas de precipitação o que se refletiu em semelhança na profundidade média ao longo do ano. As temperaturas sofreram variação sazonal típica, cujos menores valores foram encontrados nos meses de inverno. $\mathrm{O}$ oxigênio dissolvido apresentou valores elevados sendo a menor concentração registrada de 7,9 $\pm 0,4 \mathrm{mg} \mathrm{L}^{-1}$ (primavera) e a maior de 9,4 $\pm 0,7 \mathrm{mg} \mathrm{L}^{-1}$ (inverno). Os valores de turbidez, sólidos dissolvidos totais e material em suspensão, se

Tabela 3. Valores máximos dos parâmetros estabelecidos para cada Classe do CONAMA (2005)

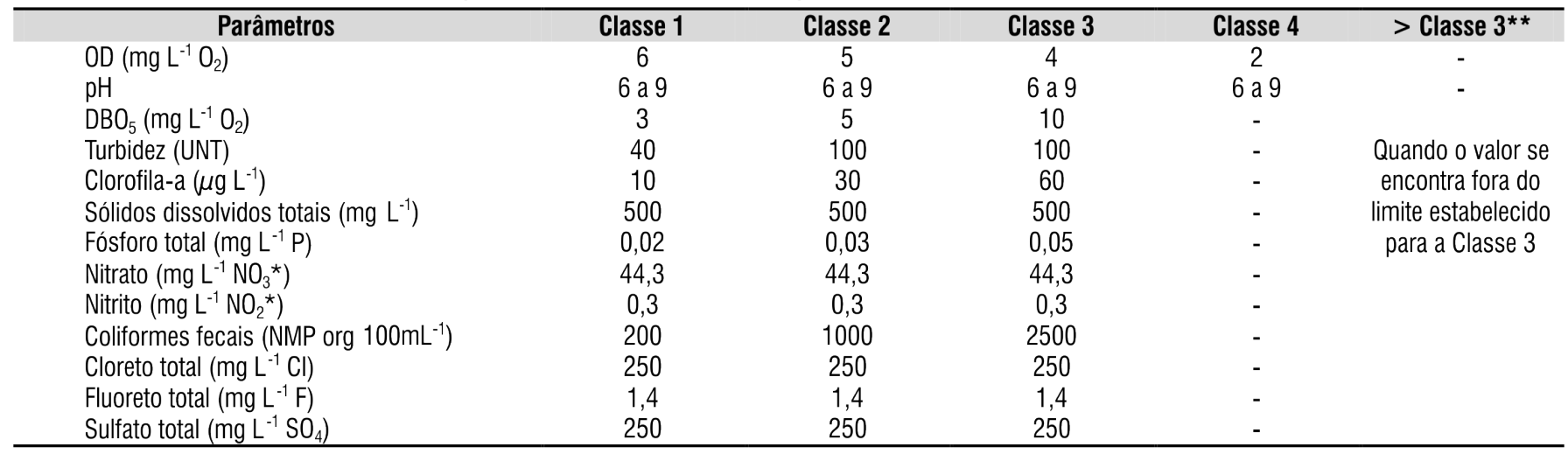

\footnotetext{
* Na Resolução 357/2005, expresso como nitrogênio (N); ** Classe incluída neste estudo
} 
Tabela 5. Médias sazonais das variáveis físico-químicas e biológicas da lagoa Caiubá. Os resultados estão expressos como média \pm desvio padrão

\begin{tabular}{|c|c|c|c|c|}
\hline Variáveis & Verão & Outono & Inverno & Primavera \\
\hline Temperatura da água $\left({ }^{\circ} \mathrm{C}\right)$ & $25,5 \mathrm{~A}$ & $22,5 \mathrm{~A}$ & 13,2 B & $19,5 \mathrm{AB}$ \\
\hline Profundidade $(\mathrm{m})$ & $2,7 \pm 0,7 \mathrm{~A}$ & $2,1 \pm 0,7 \mathrm{~A}$ & $2,6 \pm 0,6 \mathrm{~A}$ & $2,7 \pm 0,5 \mathrm{~A}$ \\
\hline Transparência (m) & $0,26 \pm 0,05 \mathrm{~A}$ & $0,23 \pm 0,05 \mathrm{AB}$ & $0,2 \pm 0,01 \mathrm{BC}$ & $0,2 \pm 0,01 \mathrm{C}$ \\
\hline Oxigênio dissolvido (mg L-1) & $8,1 \pm 0,4 \mathrm{~A}$ & $8,4 \pm 0,8 \mathrm{~A}$ & $9,4 \pm 0,7 \mathrm{~B}$ & $7,9 \pm 0,4 \mathrm{~A}$ \\
\hline $\mathrm{pH}$ & $7,3 \pm 0,3 \mathrm{~A}$ & $7,8 \pm 0,4 \mathrm{~B}$ & $7,1 \pm 0,3 \mathrm{C}$ & $7,4 \pm 0,2 \mathrm{D}$ \\
\hline Condutividade elétrica $\left(\mu \mathrm{S} \mathrm{cm}{ }^{-1}\right)$ & $83,9 \pm 4 \mathrm{~A}$ & $78,5 \pm 4 \mathrm{~A}$ & $81,2 \pm 16,2 \mathrm{~A}$ & $79,5 \pm 4,3 \mathrm{~A}$ \\
\hline Turbidez (UNT) & $107,7 \pm 10,3 \mathrm{~A}$ & $118,1 \pm 22,2 \mathrm{AB}$ & $125,7 \pm 13,3 \mathrm{BC}$ & $138,2 \pm 18,3 \mathrm{C}$ \\
\hline Alcalinidade $\left(\mu \mathrm{eq} \mathrm{L}^{-1}\right)$ & $154,5 \pm 59,6 \mathrm{~A}$ & $249,3 \pm 62,3 B$ & $169,6 \pm 46,7 \mathrm{~A}$ & $184,1 \pm 59,8 \mathrm{~A}$ \\
\hline Material em suspensão (mg L-1) & $15,8 \pm 5 \mathrm{~A}$ & $18,8 \pm 9,8 \mathrm{AB}$ & $23,0 \pm 7 \mathrm{~B}$ & $24 \pm 9,6 \mathrm{~B}$ \\
\hline Clorofila-a $\left(\mu \mathrm{g} \mathrm{L}{ }^{-1}\right)$ & $10,6 \pm 8,9 \mathrm{~A}$ & $6,8 \pm 2,2 \mathrm{AB}$ & $3,1 \pm 0,7 \mathrm{~B}$ & $4 \pm 1,1 \mathrm{~B}$ \\
\hline Sólidos dissolvidos totais $\left(\mathrm{mg} \mathrm{L}^{-1}\right)$ & $130,6 \pm 11,9 \mathrm{~A}$ & $144,3 \pm 29,2 \mathrm{AB}$ & $153 \pm 16,5 B$ & $154,9 \pm 14,6 \mathrm{~B}$ \\
\hline Fósforo total $\left(\mu \mathrm{g} \mathrm{L}^{-1}\right)$ & $123,6 \pm 29,2 \mathrm{~A}$ & $119,9 \pm 34,7 \mathrm{AB}$ & $126 \pm 12,9 \mathrm{~A}$ & $101,5 \pm 12,8 \mathrm{~B}$ \\
\hline Nitrogênio Kjedahl (mg L-1) & $0,97 \pm 0,16 \mathrm{~A}$ & $0,74 \pm 0,16 \mathrm{~B}$ & $0,73 \pm 0,26 \mathrm{~B}$ & $0,88 \pm 0,12 \mathrm{AB}$ \\
\hline Razão N:P & $8,4 \pm 3,1 \mathrm{~A}$ & $6,9 \pm 2,8 \mathrm{AB}$ & $6 \pm 2,4 \mathrm{~B}$ & $8,7 \pm 0,6 \mathrm{~A}$ \\
\hline Fosfato $\left(\mu \mathrm{g} \mathrm{L}^{-1}\right)$ & $49,5 \pm 8,1 \mathrm{~A}$ & $86,2 \pm 11 \mathrm{C}$ & $29 \pm 26,4 \mathrm{~B}$ & $44 \pm 28,7 \mathrm{AB}$ \\
\hline Nitrato $\left(\mathrm{mg} \mathrm{L}^{-1}\right)$ & $1,39 \pm 0,52 \mathrm{AB}$ & $1,1 \pm 0,37 \mathrm{AC}$ & $1,07 \pm 0,42 \mathrm{C}$ & $1,57 \pm 0,41 \mathrm{~B}$ \\
\hline Nitrito $\left(\mathrm{mg} \mathrm{L}^{-1}\right)$ & $0,005 \pm 0,002 \mathrm{~A}$ & $0,005 \pm 0,003 \mathrm{~A}$ & $0,005 \pm 0,003 \mathrm{~A}$ & $0,005 \pm 0,002 \mathrm{~A}$ \\
\hline Cloreto $\left(\mathrm{mg} \mathrm{L}^{-1}\right)$ & $15,2 \pm 3,8 \mathrm{~A}$ & $13,1 \pm 3,9 \mathrm{~A}$ & $13,7 \pm 6,2 \mathrm{~A}$ & $13,2 \pm 3,7 \mathrm{~A}$ \\
\hline Sulfato $\left(\mathrm{mg} \mathrm{L}^{-1}\right)$ & $3,17 \pm 0,83 \mathrm{~A}$ & $2,46 \pm 0,64 B$ & $2,45 \pm 1,01 \mathrm{AB}$ & $2,9 \pm 0,66 \mathrm{AB}$ \\
\hline $\mathrm{DBO}\left(\mathrm{mg} \mathrm{L}^{-1}\right)$ & $1,13 \pm 0,67 \mathrm{~A}$ & $0,60 \pm 0,16 \mathrm{~B}$ & $0,87 \pm 0,32 \mathrm{AB}$ & $0,73 \pm 0,09 \mathrm{AB}$ \\
\hline
\end{tabular}

Letras diferentes representam com diferença significativa (ANOVA, $p<0,05)$

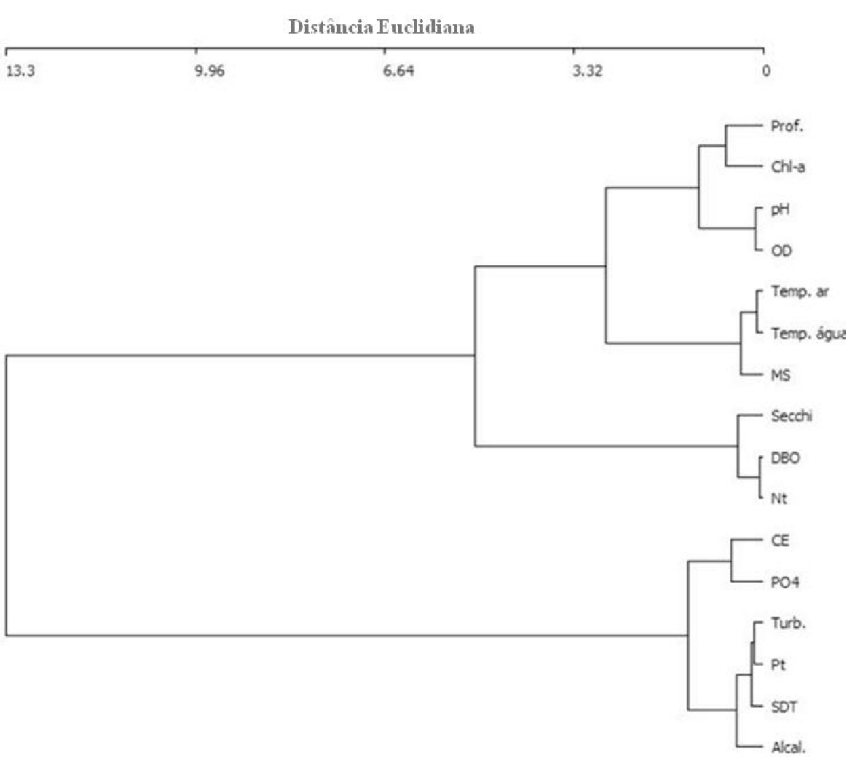

Figura 2. Dendrograma da ordenação sazonal das variáveis da água da lagoa Caiubá

apresentaram sempre com altos valores, com os maiores sendo registrados na primavera, o que se refletiu na baixa transparência da coluna de água. A extensão da zona eufótica durante o Verão, Outono, Inverno e Primavera compreendeu, em média, apenas $26 \%(0,7 \mathrm{~m}), 29,8 \%(0,63 \mathrm{~m}), 21,1 \%(0,55 \mathrm{~m})$ e $20,2 \%(0,54$ m) da profundidade, respectivamente.

Algumas variáveis mostraram forte relação com alguma estação, como clorofila-a, com aumento nos meses de verão, e $\mathrm{PO}_{4}$ com aumento nos meses de outono. Entre os íons analisados as variações entre cloretos e sulfatos foram marcantes. As concentrações de cloreto na lagoa Caiubá foram baixas e não variaram temporalmente indicando baixa salinidade e pouca ou quase nenhuma influência marinha; já o íon sulfato teve sua maior concentração no verão coincidindo com o período de produção de arroz. Entre as fontes que podem influir na distribuição deste elemento nos ecossistemas aquáticos, está a aplicação de adubos contendo enxofre em áreas agrícolas.

Os valores de nutrientes se caracterizaram pelas baixas concentrações, com exceção do fósforo, que apresentou valores elevados durante todo o ano. Esta alta taxa de fósforo pode ser um indicativo de enriquecimento através das atividades no entorno, como evidenciado em vários estudos de degradação de lagos rasos por atividades agrícolas (Nakano et al., 2008; James et al., 2009). As baixas concentrações de nitrogênio fizeram com que a razão $\mathrm{N}: \mathrm{P}$ se mantivesse em valores baixos ao longo do ano e em todas as regiões da lagoa, caracterizando este sistema com sua produtividade limitada pelas concentrações de nitrogênio (Figura 3).

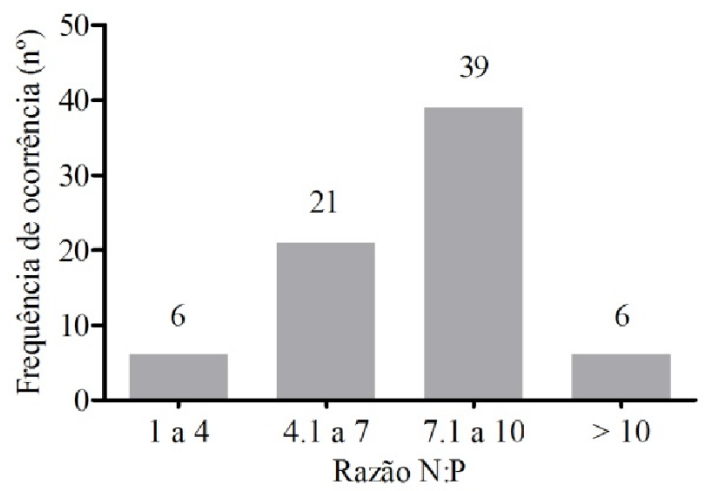

Figura 3. Distribuição de frequência dos valores da razão N:P em todas as amostras da lagoa Caiubá, durante dezembro de 2009 e novembro de 2010, $(n=72)$

Kratzer \& Brezonik (1981) estudando lagos rasos tropicais na Flórida consideraram ambientes com produtividade limitada por nitrogênio, aqueles que apresentam razão $\mathrm{N}: \mathrm{P}$ menor que 10. Os resultados desta pesquisa indicaram que apenas em seis das amostragens esses valores ultrapassaram tal valor, podendo a lagoa Caiubá ser considerada limitada por nitrogênio. De forma geral, os maiores valores foram encontrados nas coletas 
de verão e outono ocorrendo uma diminuição a partir do inverno e primavera. A baixa razão N:P em lagos rasos subtropicais é capaz de promover uma situação de limitação secundária por nitrogênio, que ocorre quando o nível de fósforo é aumentado para níveis excedentes ou quando há uma diminuição das concentrações de nitrogênio (James et al., 2009). A queda do nitrogênio na água pode ser devida ao término da safra do arroz diminuindo os resíduos que a lixiviação das plantações leva para a lagoa. Esta situação merece atenção pois já foi constatado que lagos rasos com altas concentrações de fósforo e baixa razão $\mathrm{N}: \mathrm{P}$ são ambientes favoráveis a florações de cianobactérias (Havens et al., 2003).

Os valores encontrados para concentração de clorofila-a demonstram a baixa produtividade da lagoa Caiubá. Embora os valores de concentração de clorofila-a tenham apresentado leve incremento no verão, em nenhuma das estações do ano ficou evidenciado crescimento excessivo do fitoplâncton, haja vista que os máximos valores médios atingiram 10,6 $\pm 8,9 \mu \mathrm{g} \mathrm{L}^{-1}$ mas, conforme as categorizações dos índices de estado trófico (Tabela 2), em valores de clorofila maiores que $11 \mu \mathrm{g} \mathrm{L}^{-1}, \mathrm{o}$ ambiente já pode ser classificado como de moderada qualidade.

A mistura da coluna de água visualizada pelos perfis homogêneos de oxigênio e a temperatura ao longo do ano, demonstram a característica polimítica do ecossistema. A lagoa Caiubá apresentou altos valores de turbidez, sólidos dissolvidos e material em suspensão, o que ocasionou os baixos valores de transparência. Pelos baixos valores de clorofila-a esta turbidez é devida ao material inorgânico carreado através de lixiviação do entorno e à ação do vento ressuspendendo o sedimento.

O IET é uma medida do grau de trofia dos corpos de água largamente utilizado em sistemas aquáticos em todo o mundo. Poucos estudos utilizando o IET foram realizados em lagoas costeiras no Rio Grande do Sul, dentre os quais Schäfer (1988) cita, através de uma modificação no índice original de Carlson (1977), que o maior índice foi encontrado para a lagoa Caiubá e ressalta, ainda, que entre 38 lagoas estudadas aquelas mais rasas são as que apresentaram os maiores IETs; enfim, os resultados do IET obtidos nesta pesquisa são apresentados nas Tabelas 6 e 7.
Com exceção dos valores de clorofila, em que os enquadramentos de qualidade de água apresentaram algumas estações do ano com valores caracterizando oligotrofia, todos os outros oscilaram entre hipereutrófico a eutrófico. Apesar dos baixos valores de clorofila-a encontrados, os resultados da concentração de fósforo total e transparência podem ser os responsáveis pela classificação da lagoa Caiubá como um corpo de água eutrófico, durante a maior parte do ano. A condição mais eutrofizada da lagoa no inverno pode ser devida à influência das culturas de arroz no período anterior fornecendo uma carga externa de nutrientes e resíduos para a lagoa.

Em pesquisa da qualidade de água da bacia da Lagoa Mirim, Fia et al. (2009) encontraram situação semelhante para os corpos de água lênticos da região. Segundo os autores, aplicando a metodologia de Lamparelli (2004) todos os corpos de água lênticos foram classificados como eutróficos e hipereutróficos. Estes resultados foram atribuídos às características do entorno dos corpos de águas. Alguns corpos hídricos são inseridos em regiões de intensa atividade agrícola, que apresentam lavouras de arroz irrigado, como a lagoa Caiubá, que os utiliza para captação de água com fins à irrigação de suas lavouras. A devolução da água utilizada nas lavouras de arroz com resíduos de adubos químicos e orgânicos fosfatados estabelece uma contribuição difusa ao longo deles (Grützmacher et al., 2008) podendo explicar os valores de IET encontrados durante o período de observação (Fia et al., 2009).

A turbidez provocada pela lixiviação do material de lavouras de arroz associada com a baixa profundidade da lagoa e o regime de ventos constantes na região promovem a ressuspensão do sedimento contribuindo, sem dúvida, para recolocar em circulação os nutrientes, sobretudo o fósforo, já que este é outro componente do cálculo do IET e suas concentrações na lagoa Caiubá estão em faixas preocupantes no tocante à possibilidade de favorecimento de florações algais.

Os índices de qualidade de água são representados por um valor numérico que traduz, mesmo resumidamente, a qualidade de água, sendo largamente utilizados em monitoramento de recursos hídricos (Bruschi Júnior et al., 1998). Baseado no índice desenvolvido pela National Sanitation Foundation, dos

Tabela 6. Resultado dos Índices de Estado Trófico modificado por Lamparelli (IET) calculados sazonalmente e para todo o período amostral, entre dezembro de 2009 e novembro de 2010, para a lagoa Caiubá

\begin{tabular}{|c|c|c|c|c|c|c|c|c|c|c|}
\hline Estação & $\mathrm{IET}_{\mathrm{L}}$ (Clo) & Enq. & IET $_{\mathrm{L}}\left(\mathrm{P}_{\mathrm{T}}\right)$ & Enq. & $\mathrm{IET}_{\mathrm{L}}$ (Sec) & Enq. & IET $_{\mathrm{L}}$ Geral & Enq. & $\mathrm{IET}_{\mathrm{L}}\left(\mathrm{P}_{\mathrm{T}} \mathrm{eCl} 0\right)$ & Enq. \\
\hline Inverno & 49 & oligo & 64 & super & 76 & hiper & 63 & super & 56 & meso \\
\hline Geral Anual & 55 & meso & 63 & super & 75 & hiper & 63 & super & 74 & hiper \\
\hline
\end{tabular}

* Enq - enquadramento; IET $(\mathrm{Clo})$ - com os valores de clorofila; IET $\left(\mathrm{P}_{\mathrm{T}}\right)$ - com os valores de fósforo total; IET $(\mathrm{Sec})$ - com os valores de transparência; IET $G$ Geral - com as médias dos valores; hiper -hipereutrófico; super -supereutrófico; eut -eutrófico; meso -mesotrófico;oligo -oligotrófico

Tabela 7. Resultado dos Índices de Estado Trófico modificado por Mercante e Moura (IET ${ }_{M}$ ) calculados sazonalmente e para todo o período amostral, entre dezembro de 2009 e novembro de 2010, para a lagoa Caiubá

\begin{tabular}{|c|c|c|c|c|c|c|c|c|c|c|}
\hline Estação & $\mathrm{IET}_{\mathrm{M}}$ (Clo) & Enq. & $\operatorname{IET}_{M}\left(P_{T}\right)$ & Enq. & $\mathrm{IET}_{\mathrm{M}}(\mathrm{Sec})$ & Enq. & $\mathrm{IET}_{M}$ (FSR) & Enq. & IET $_{M}$ Geral & Enq. \\
\hline Verão & 54 & eut & 66 & eut & 70 & eut & 72 & eut & 47 & meso \\
\hline Outono & 50 & meso & 65 & eut & 71 & eut & 80 & eut & 65 & eut \\
\hline Inverno & 35 & oligo & 66 & eut & 74 & eut & 64 & eut & 72 & eut \\
\hline Primavera & 39 & oligo & 63 & eut & 74 & eut & 70 & eut & 73 & eut \\
\hline Geral Anual & 47 & meso & 65 & eut & 72 & eut & 73 & eut & 63 & eut \\
\hline
\end{tabular}

* Enq - enquadramento; IET $\left(\mathrm{Clo}\right.$ - com os valores de clorofila; $\mathrm{IET}_{M}\left(\mathrm{P}_{\mathrm{T}}\right)$ - com os valores de fósforo total; IET (Sec) - com os valores de transparência; IET (FSR) - com os valores de fósforo solúvel reativo; $\mathrm{IET}_{\mathrm{M}}$ Geral - com as médias ponderadas; eut -eutrófico;meso -mesotrófico;oligo -oligotrófico 
Tabela 8. Variação espacial e sazonal do Índice de Qualidade da Água (IQA) da lagoa Caiubá

\begin{tabular}{|c|c|c|c|c|c|c|c|c|}
\hline \multirow{2}{*}{ Parte } & \multicolumn{2}{|c|}{ Verão } & \multicolumn{2}{|c|}{ Outono } & \multicolumn{2}{|c|}{ Inverno } & \multicolumn{2}{|c|}{ Primavera } \\
\hline & IQA & Qualidade & IQA & Qualidade & IQA & Qualidade & IQA & Qualidade \\
\hline Norte & 90 & Boa & 79 & Boa & 86 & Boa & 89 & Boa \\
\hline Centro & 94 & Excelente & 86 & Boa & 96 & Excelente & 96 & Excelente \\
\hline Sul & 97 & Excelente & 85 & Boa & 98 & Excelente & 98 & Excelente \\
\hline Média & 94 & Excelente & 83 & Boa & 93 & Excelente & 94 & Excelente \\
\hline
\end{tabular}

Estados Unidos, o Comitê de Gerenciamento da Bacia do Rio dos Sinos (COMITESINOS) desenvolveu adaptações passíveis de promover sua aplicação para o rio dos Sinos, no Rio Grande do Sul. Este índice tem sido aplicado no monitoramento de vários corpos hídricos no estado (Blume et al., 2010). O IQA classificou as águas da lagoa Caiubá no Verão, Inverno e Primavera, como de "excelente" qualidade e no Outono como de "boa" qualidade (Tabela 5), representando uma água propícia para abastecimento humano; além disto, no verão, inverno e na primavera foi evidenciada uma melhora na qualidade da água, nas suas partes central e sul (Tabela 8).

Segundo Piasentin et al. (2009), o IQA reflete principalmente a contaminação dos corpos hídricos, causada pelo lançamento de esgotos domésticos. Lopes et al. (2008) chamam a atenção, através de análises isoladas das variáveis que compõem o IQA, para o fato de que o valor isolado deste índice não é suficiente para uma análise acurada da qualidade de água; segundo os autores, as oscilações das variáveis do IQA se compensam umas às outras mantendo o índice relativamente estável em um patamar, embora esta relativa "estabilidade" mascare flutuações importantes no ambiente, que devem ser monitoradas e analisadas com maior cuidado.

Um ambiente pode enquadrar-se na faixa "ótima" do IQA mesmo que haja algumas substâncias em concentrações tóxicas à biota (hidrocarbonetos e metais pesados) (Lopes et al., 2008). Em um trabalho com a lagoa do Apodi, no Rio Grande do Norte, Pinto Filho et al. (2012) também ressaltam a necessidade de avaliação isoladamente das variáveis para estabelecimento da qualidade de água uma vez que, sozinho, o IQA não contempla contaminantes potenciais importantes, como os defensivos agrícolas.

Os resultados mostram que a maioria dos parâmetros analisados da lagoa Caiubá (cerca de $80 \%$ ) se enquadra nas determinações da Classe 1 mas também apresentou alguns parâmetros, como Classes 2, 3 e > Classe 3 (Figura 4).

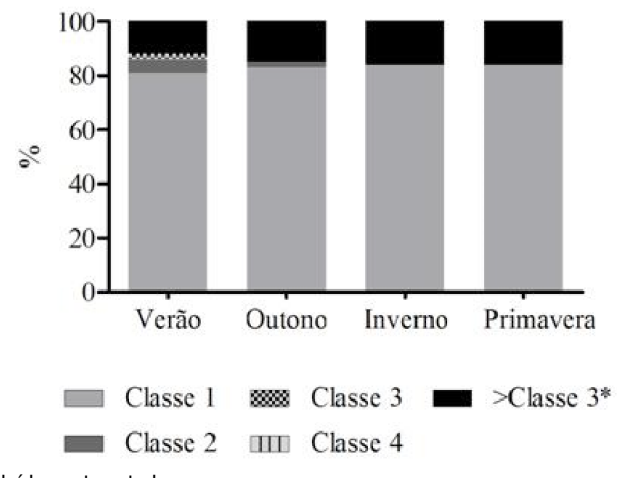

* Classe incluída neste estudo

Figura 4. Variação sazonal na proporção de classificação das variáveis físicas, químicas e biológicas da lagoa Caiubá segundo CONAMA (2005)
O verão apresentou a parte norte da lagoa com melhor qualidade do que os outros pontos em virtude da maior porcentagem de variáveis ter se enquadrado nas Classes 1 e 2 e menor porcentagem na classe $>$ Classe 3 (Figura 5).
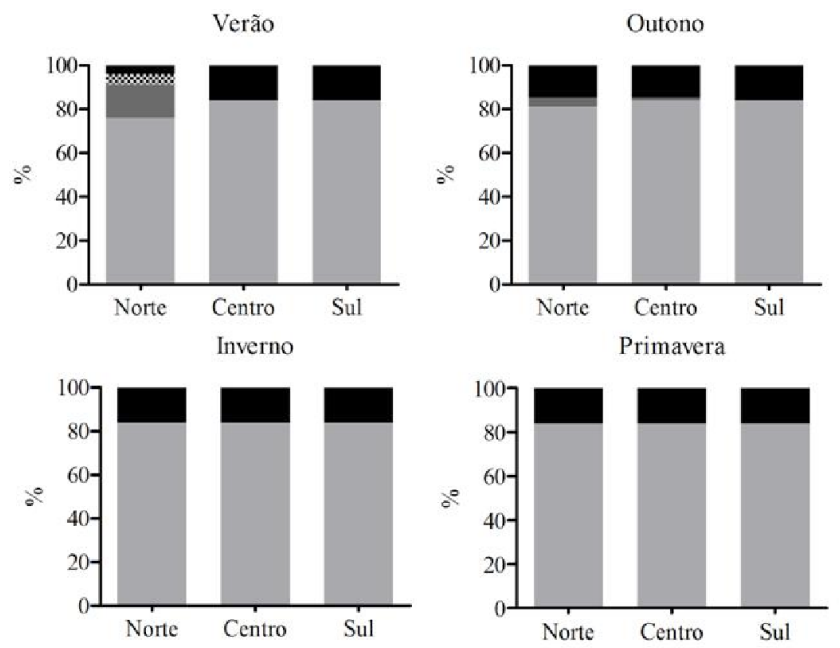

$\square$ Classe $1 \square$ Classe

* Classe incluída neste estudo

Figura 5. Variação espacial na proporção de classificação das variáveis físicas, químicas e biológicas da lagoa Caiubá para cada estação do ano segundo CONAMA (2005)

A Figura 6 mostra a frequência de ocorrência de cada variável da lagoa Caiubá distribuída nas classes do CONAMA (2005) por estação do ano.

De acordo com CONAMA (2005) a classificação do corpo hídrico é definida pelos usos preponderantes mais restritivos da água, atuais ou pretendidos. Como os principais usos que ocorrem na lagoa Caiubá são para irrigação do arroz, dessedentação de animais e para pesca, ela deve apresentar-se, no máximo, como Classe 2; segundo os resultados encontrados, a maioria dos parâmetros analisados da lagoa Caiubá (cerca de $80 \%$ ) se enquadra nas determinações da Classe 1 mas também apresentou alguns parâmetros como Classes 2, 3 e $>$ Classe 3 .

O Verão foi a estação que apresentou melhor qualidade da água em razão da maior proporção dos parâmetros até a Classe 2 e menor proporção na $>$ Classe 3 . A proporção dos parâmetros que se enquadraram na Classe 1 vai aumentando do Verão para a Primavera, o que poderia indicar uma melhora na qualidade da água ao longo do ano após o final da colheita do arroz.

Já foi evidenciado que os limites impostos pelo CONAMA (2005) são considerados muito tolerantes, principalmente para o nitrato e o nitrito (Silva et al., 2008). Os parâmetros que contribuíram para a perda da qualidade da água na lagoa Caiubá foram a turbidez, a clorofila-a e o fósforo total pela grande 


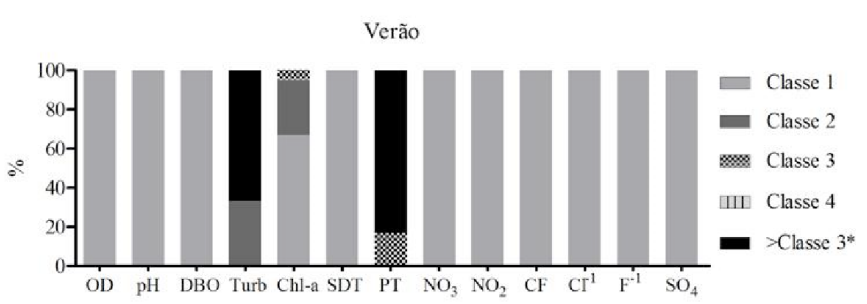

Outono

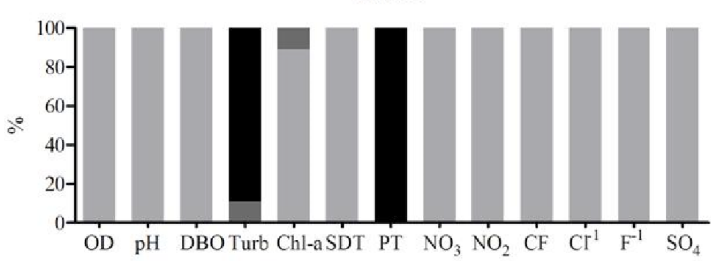

Inverno

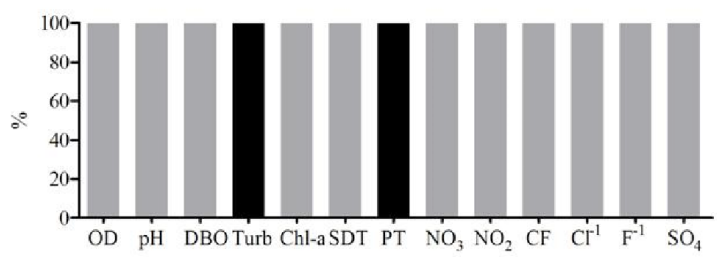

Primavera

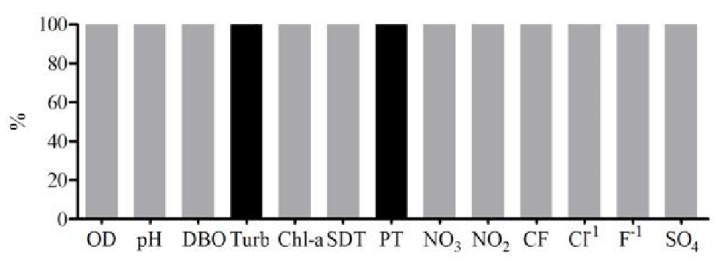

* Classe incluída neste estudo

Figura 6. Frequência de ocorrência de cada variável física, química e biológica da lagoa Caiubá nas classes do CONAMA (2005) por estação do ano (OD - oxigênio dissolvido; $\mathrm{DBO}$ - demanda bioquímica de oxigênio; Turb- turbidez; Chl-a -clorofila-a; SDT - sólidos dissolvidos totais; PT - fósforo total; $\mathrm{NO}_{3}$ - nitrato; $\mathrm{NO}_{2}$ - nitrito; $\mathrm{CF}$ - coliformes fecais; $\mathrm{Cl}^{-1}$ - cloreto; $\mathrm{F}^{-1}$ - fluoreto; $\mathrm{SO}_{4}$ - sulfato)

porcentagem considerada como acima dos valores estabelecidos pela legislação mostrando uma tendência de eutrofização desta lagoa. A ocorrência de valores acima dos limites recomendados pela legislação já foi constatada para outros corpos de água brasileiros (Silva \& Sacomani, 2001; Silva et al., 2008).

O CONAMA (2005) não determina como interpretar os dados e fazer a classificação do corpo hídrico quando as variáveis se encontram dentro de diferentes Classes. Se fosse levada em consideração a ocorrência de algumas variáveis fora dos limites estabelecidos, as águas da lagoa estariam fora dos padrões para seus usos atuais porém cerca de $80 \%$ das variáveis se encontraram na Classe 1 durante o período estudado.

Foram identificados 3363 organismos pertencentes a 19 táxons, dentre os quais 51\% foram Protozoa (Arcellidae), $21 \%$ Nematoda e 7\% Oligochaeta (Naididae) compreendendo 79\% dos organismos. Não ocorreu diferença significativa entre a composição e a abundância entre as estações do ano optandose por calcular os índices gerais (ANOVA, gl-3, F =0,247, $\mathrm{p}=$ 0,122 ). Aplicados os índices bióticos o BMWP adaptado atingiu valor de 58, em que se enquadra a lagoa Caiubá, segundo os critérios da Secretaria de Meio Ambiente e Recursos Hídricos do Paraná como água de qualidade duvidosa. Resultado semelhante é encontrado quando se aplica o índice biótico da EPA, que atingiu valor de 8,4, sendo enquadrada na classe IV, de qualidade ruim; de acordo com a adaptação de Junqueira \& Campos (1998) e os critérios utilizados pela CETEC de Minas Gerais, a lagoa Caiubá pode ser enquadrada como águas de qualidade satisfatória.

Ao contrário dos índices de qualidade de água e do enquadramento na legislação brasileira, os resultados dos índices bióticos demonstraram uma situação de águas de baixa qualidade. Os valores e organismos predominantes encontrados são comuns em ambientes alterados com características marcantes de contaminação por esgotos domésticos e/ou poluição de origem agrícola ou industrial. A relação dos resultados obtidos é maior com os valores de IET ao longo do período de estudo, resultado também encontrado por Rossaro et al. (2007) em lagos italianos.

O IQA e IET mostraram algumas alterações ao longo do ano, provavelmente reflexo do maior uso da lagoa nos períodos de cultivo do arroz. Sabe-se que as medidas de parâmetros químicos e físicos são pontuais refletindo o momento em que são medidos. Analisando a estrutura da comunidade de invertebrados obtém-se, ao contrário, um resultado que reflete o acúmulo de condições pelas quais o ambiente passou. Desta forma e através da avaliação da comunidade bentônica, os indicativos são de que a lagoa Caiubá se encontra em situação preocupante quanto à qualidade de suas águas.

Os resultados comprovam a fragilidade de estabelecimento de qualidade de água quando a fonte poluidora não é constituída por esgotos domésticos e ressaltam, também, a necessidade de adequações para ecossistemas aquáticos impactados por fontes difusas de entrada de compostos que alteram a qualidade de água, como agricultura e pecuária.

A inserção deste ecossistema no sistema hidrológico do Taim e as características da planície costeira sul, que interligam seus ecossistemas aquáticos, ressaltam a preocupação no controle e manutenção dos ecossistemas aquáticos regionais. A necessidade de análises mais detalhadas em relação a compostos não contemplados neste estudo, como defensivos agrícolas e resíduos de agricultura, tornam-se imperativos para o uso sustentável deste ecossistema visando à manutenção de sua qualidade e biodiversidade.

Assim, sugere-se a reavaliação dos parâmetros utilizados na avaliação de qualidade de água e utilização da análise das variáveis medidas quando a fonte poluidora do ecossistema aquático tiver origem em atividades agropecuárias e não em esgotos domésticos.

\section{Conclusões}

1. O estudo das variáveis limnológicas evidenciou que a lagoa Caiubá se encontra num estado de águas túrbidas, mantida pela constante ressuspensão do sedimento e não pela produção algal. A lagoa apresentou valores de fósforo total e turbidez acima dos padrões estipulados pelo CONAMA, durante todo o ano estudado. 
2. A aplicação dos índices para avaliar a qualidade da água teve resultados contrastantes: enquanto o índice de estado trófico e os índices bióticos demonstraram que a lagoa Caiubá se encontra eutrofizada, com baixa qualidade de água, os resultados do índice de qualidade de água classificaram a lagoa com boa a excelente qualidade e cerca de $80 \%$ dos parâmetros se enquadraram na Classe 1 (águas de melhor qualidade) pela legislação CONAMA.

\section{Agradecimentos}

Os autores agradecem à Universidade Federal do Rio Grande (FURG), à Coordenação de Aperfeiçoamento de Pessoal de Nível Superior (CAPES), à Fundação de Amparo à Pesquisa do Estado do Rio Grande do Sul (FAPERGS) (905418), à Granja Quatro Irmãos e ao Instituto Chico Mendes da Conservação da Biodiversidade (ICMBio) pelo apoio ao trabalho.

\section{Literatura Citada}

Alba, J. M. F.; Schroder, V. F.; Nóbrega, M. R. Land cover change detection in Southern Brazil through orbital imagery classification methods. In: Escalante, B. Remote Sensing Applications. Rijeka: INTECH, 2012, chapter 4. p.99-116.

Blume, K. K.; Macedo, J. C.; Meneguzzi, A.; Silva, L. B.; Quevedo, D. M.; Rodrigues, M. A. S. Water quality assessment of the Sinos River, Southern Brazil. Brazilian Journal of Biology, v.70, p.1185-1193, 2010.

Bruschi Júnior, W.; Salomoni, S. E.; Fermino, F. S.; Pedrozo, C. S.; Schwarzbold, A.; Peret, A. C. Aplicação de um índice de qualidade de água para lagoas costeiras, RS, afetadas por despejos urbanos. Biociências, v.6, p.55-66, 1998.

Carlson, R.E. A trophic state index for lakes. Limnology and Oceanography, v.22, p.361-369,1977.

COMITESINOS - Comitê de Gerenciamento da Bacia Hidrográfica do Rio dos Sinos. Utilização de um Índice de Qualidade da Água para o Rio dos Sinos/RS. Porto Alegre: COMITESINOS, $1990.33 \mathrm{p}$.

CONAMA - Conselho Nacional do Meio Ambiente. Resolução no 357, de 17 de março de 2005. Dispõe sobre a classificação dos corpos de água e diretrizes ambientais para o seu enquadramento, bem como estabelece as condições e padrões de lançamento de efluentes, e dá outras providências. Brasília: DOU, 2005. 23p.

Coveney, M. F.; Lowe, E. F; Battoe, L. E.; Marzolf, E. R.; Conrow, R. Response of a eutrophic, shallow subtropical lake to reduced nutrient loading. Fresh Water Biology, v.50, p.1718-1730, 2005.

Fia, R.; Matos, A. T.; Coradi, P. C.; Pereira-Ramirez, O. Estado trófico da água na bacia hidrográfica da Lagoa Mirim, RS, Brasil. Revista Ambiente \& Água - An Interdisciplinary Journal of Applied Science, v.4, p.132-141, 2009.

Grützmacher, D. D.; Grützmacher, A. D.; Agostinetto, D.; Loeck, A. E.; Roman, R.; Peixoto, S. C.; Zanella, R. Monitoramento de agrotóxicos em dois mananciais hídricos no sul do Brasil. Revista Brasileira de Engenharia Agrícola e Ambiental, v.12, p.632-637, 2008.

Hammer, O.; Harper D. A. T.; Ryan, P. D. Past-Paleontological statistics software package for education and data analysis. Paleontologia Electronica, v.4, p.1-9, 2001.
Havens, K. E.; James, R. T.; East, T. L.; Smith, V. H. N:P ratios, light limitation, and cyanobacterial dominance in a subtropical lake impacted by non-point source nutrient pollution. Environmental Pollution, v.122, p.379-390, 2003.

James, R. T.; Havens, K.; Zhu, G.; Qin, B. Comparative analysis of nutrients, chlorophyll and transparency in two large shallow lakes (Lake Taihu, P. R. China and Lake Okeechobee, USA). Hydrobiologia, v.627, p.211-231,2009.

Janse, J. H.; Domis, L. N. S. de; Scheffer, M.; Lijklema, L.; van Liere, L.; Klinge, M.; Mooij, W. M. Critical phosphorus loading of different types of shallow lakes and the consequences for management estimated with the ecosystem model PCLake. Limnologica,v.38, p.203-219, 2008.

Junqueira, V. M.; Campos, S. C. M. Adaptationofthe "BMWP" method for water quality evaluation to Rio das Velhas watershed (Minas Gerais, Brazil). Acta Limnologica Brasiliensia, v.10, p.125-135, 1998.

Kratzer, C. R.; Brezonik, P. L. A Carlson-type trophic state index for nitrogen in Florida lakes. Water Resources Bulletin, v.17, p.713-715, 1981.

Lamparelli, M. C. Graus de trofia em corpos de água do estado de São Paulo: avaliação dos métodos de monitoramento. São Paulo: USP, 2004. 238p. Tese Doutorado

Lopes, F. B.; Teixeira, A. S.; Andrade, E. M.; Aquino, D. N.; Araújo, L. F. P. Mapa da qualidade das águas do rio Acaraú, pelo emprego do IQA e geoprocessamento. Revista Ciência Agronômica, v.39, p.392-402,2008.

Mercante, C. T. J.; Moura, A. T. Comparação Entre os índices de Carlson e de Carlson modificado aplicados a dois ambientes aquáticos subtropicias, São Paulo, SP. Acta Limnologica Brasiliensia, v.12, p.1-21, 1999.

Nakano, T.; Tayasu, I.; Yamada, Y.; Hosono, T.; Igeta, A.; Hyodo, F.; Ando, A.; Saitoh, Y.; Tanaka, T.; Wada, E.; Yachi, S. Effect of agriculture on water quality of Lake Biwa tributaries, Japan. Science ofthe Total Environment, v.389, p.132-148, 2008.

Piasentin, A. M.; Semensatto Júnior, D. L.; Saad, A. R.; Monteiro Júnior, A. J.; Raczka, M. F. Índice de qualidade da água (IQA) do reservatório Tanque Grande, Guarulhos (SP): Análise sazonale efeitos do uso e ocupação do solo. Geociências, v.28, p.305-312, 2009.

Pinto Filho, J. L. O.; Santos, E. G.; Souza, M. J. J. B. Proposta de índice de qualidade de água para a Lagoa do Apodi, RN, Brasil. Holos, v.28, p.69-76, 2012.

Rosenberg, D. M.; Resh, V. H. (ed.). Fresh water biomonitoring in benthicmacro invertebrates. Chapman \& Hall: New York, 1993. 488p.

Rossaro, B.; Marziali, L.; Cardoso, A. C.; Solimini, A. G; Free, G.; Giacchini, R. A biotic index using benthicmacroin vertebrates for Italian lakes. Ecological Indicators, v.7, p.412-429, 2007.

Schäfer,A. Tipificação ecológica de lagoas costeiras do Rio Grande do Sul. Acta Limnologica Brasiliensia, v.2, p.29-55, 1988.

Scheffer, M. Ecology of shallow lakes. Population and community biology series. Chapman \& Hall: Londres, 1998. 357p.

Silva, A. M. M. da; Sacomani, L. B. Using chemical and physical parameters to define the quality of Pardo river water (Botucatu-SP-Brazil). Water Research, v.35, p.16091616, 2001. 
Silva, D. M.; Pereira, W. C.; Kligerman, D. C.; Jacob, S. C. Evaluation of the contaminating potential of an important tributary which flows in to Guanabara Bay, Rio de Janeiro, Brazil. Management of Environmental Quality: An International Journal, v.19, p.20-36, 2008.

Trindade, C. R. T.; Furlanetto, L. M.; Silva, C. P. Nycthemeral cycles and seasonal variation of limnological factors of a subtropical shallow lake (Rio Grande, RS, Brazil). Acta Limnologica Brasiliensia, v.21, p.35-44, 2009.
Zambrano, L.; Contreras, V.; Hiriart, M. M.; Arista, A. E. Z. Spatial heterogeneity of water quality in a highly degraded tropical freshwater ecosystem. Environmental Management, v.43, p.249-263, 2009.

Zhu, G.; Qin, B.; Gao, G.; Zhang, L.; Luo, L.; Zhang, Y. Effects of hydrodynamics on phosphorus concentrations in water of lake Taihu, a large, shallow, eutrophic lake of China. Hydrobiologia, v.581, p.53-61, 2007. 